\title{
A genomic screen for angiosuppressor genes in the tumor endothelium identifies a multifaceted angiostatic role for bromodomain containing 7 (BRD7)
}

\author{
Judy R. van Beijnum ${ }^{1}$ Patrycja Nowak-Sliwinska ${ }^{2}$ - Maaike van Berkel ${ }^{1}$ \\ Tse J. Wong ${ }^{1}$ - Arjan W. Griffioen ${ }^{1}$
}

Received: 26 June 2017 / Accepted: 12 September 2017 / Published online: 26 September 2017

(C) The Author(s) 2017. This article is an open access publication

\begin{abstract}
Tumor angiogenesis is characterized by deregulated gene expression in endothelial cells (EC). While studies until now have mainly focused on overexpressed genes in tumor endothelium, we here describe the identification of transcripts that are repressed in tumor endothelium and thus have potential suppressive effects on angiogenesis. We identified nineteen putative angiosuppressor genes, one of them being bromodomain containing 7 (BRD7), a gene that has been assigned tumor suppressor properties. BRD7 was studied in more detail, and we demonstrate that BRD7 expression is inversely related to EC activation. Ectopic expression of BRD7 resulted in a dramatic reduction of EC proliferation and viability. Furthermore, overexpression of BRD7 resulted in a bromodomain-dependent induction of NFkB-activity and NFkB-dependent gene expression, including ICAM1, enabling leukocyte-endothelial interactions. In silico functional annotation analysis of genomewide expression data on BRD7 knockdown and overexpression revealed that the transcriptional signature of low BRD7 expressing cells is associated with increased angiogenesis (a.o. upregulation of angiopoietin-2, VEGF receptor-1 and neuropilin-1), cytokine activity (a.o. upregulation of CXCL1
\end{abstract}

Electronic supplementary material The online version of this article (doi:10.1007/s10456-017-9576-3) contains supplementary material, which is available to authorized users.

Arjan W. Griffioen

aw.griffioen@vumc.nl

http://www.angiogenesis.nl

1 Angiogenesis Laboratory, Department of Medical Oncology, Cancer Center Amsterdam, VU University Medical Center, De Boelelaan 1117, 1081 HV Amsterdam, The Netherlands

2 School of Pharmaceutical Sciences, University of Geneva (UNIGE), Geneva, Switzerland and CXCL6), and a reduction of immune surveillance (TNF$\alpha$, NFKB, ICAM1). Thus, combining in silico and in vitro data reveals multiple pathways of angiosuppressor and antitumor activities of BRD7.

Keywords BRD7 - Angiogenesis - Tumor endothelial cells $\cdot$ Gene expression $\cdot$ CXCL1 $\cdot$ NFkB

$\begin{array}{ll}\text { Abbreviations } \\ \text { EC } & \text { Endothelial cell } \\ \text { BRD7 } & \text { Bromodomain containing } 7 \\ \text { TEC } & \text { Tumor EC } \\ \text { NEC } & \text { Normal EC } \\ \text { PLEC } & \text { Placenta EC } \\ \text { HUVEC } & \text { Human umbilical vein endothelial cell } \\ \text { qPCR } & \text { Quantitative real-time PCR } \\ \text { CAM } & \text { Chicken chorioallantoic membrane } \\ \text { VEGF } & \text { Vascular endothelial growth factor } \\ \text { TKI } & \text { Tyrosine kinase inhibitor } \\ \text { TNF- } \alpha & \text { Tumor necrosis factor- } \alpha \\ \text { NFkB } & \text { Nuclear factor kappa B } \\ \text { ICAM1 } & \text { Intercellular adhesion molecule 1 } \\ \text { CXCL } & \text { Chemokine (C-X-C motif) ligand } \\ \text { XAF1 } & \text { XIAP-accociated factor 1 } \\ \text { XIAP } & \text { X-linked inhibitor of apoptosis } \\ \text { PBAF } & \text { Polybromo-associated BAF } \\ \text { BAF } & \text { BRG1-associated factor } \\ \text { BRG1 } & \text { Brahma-related gene 1 } \\ & \end{array}$

\section{Introduction}

Angiogenesis is pivotal to the progression of cancer, and inhibition of this process is considered a promising therapeutic strategy [1]. Clinical success of angiogenesis inhibition, 
however, is still rather limited, and problems associated with toxicity and acquired or inherent resistance to therapy are emerging. Moreover, therapeutic inhibition of the VEGF/ VEGFR signaling axis was suggested to accelerate the formation of distant metastases (reviewed in [2]). We believe it is therefore necessary to gain more insight in endothelial cell (EC) biology to identify more relevant targets and design better angiostatic therapeutics.

Tumor angiogenesis is characterized by deregulated gene expression in EC, which contributes to enhanced proliferation, tube formation and matrix remodeling, to comply with the functional demands of the growing tumor. In recent years, technological improvements have facilitated the use of purified cell populations for molecular studies [3, 4], and an increasing number of putative markers of endothelium in diverse tumors have been reported $[3,5]$. These markers can be targeted for therapeutic and diagnostic applications as we have demonstrated previously $[3,6,7]$, and have direct clinical relevance.

In contrast to tumor cell biology, where both positive regulators (oncogenes) and negative regulators (tumor suppressor genes) have been extensively studied, focus in the field of angiogenesis has been predominantly on overexpression of genes because of targeting potential. Studies analyzing gene silencing in tumor EC are sparse and have mainly been performed in the context of promoter methylation [8-10]. In the current study, we have performed a gene expression analysis in EC of tumor- and normal tissues and queried for transcripts specifically downregulated in tumor endothelium.

We found 19 unique transcripts that were downregulated in colon tumor EC as compared to both normal colon EC and placenta EC. Of these, only very few have a reported association with cancer and/or angiogenesis. A notable exception was bromodomain containing 7 (BRD7) that triggered our interest by its previously proposed tumor suppressor function in different types of cancer [11-14].

Bromodomain proteins are a large family of chromatin binding molecules that are highly conserved through evolution. BRD7 is a subunit of the PBAF-specific SWI/SNF chromatin remodeling complex $[15,16]$. It binds to acetylated histones and serves to modulate transcription factor activity $[15,17]$. Recently, it was shown that BRD7 binds directly to p53 and is required for efficient transcription of a subset of p53 target genes, such as p21 and PAI-1 [11, 18]. In addition, direct binding of BRD7 to BRCA1 was demonstrated [15]. Furthermore, XAF1, another transcriptional target of BRD7, is involved in endothelial cell senescence [19].

In this study, we investigated the role of BRD7 in tumor angiogenesis. We demonstrate an inverse relation between BRD7 expression levels and activation status of EC, both in vitro and in vivo. We show that suppression of BRD7 induces a pro-angiogenic cytokine and gene expression profile, whereas increased expression of BRD7 induces
NFkB-mediated ICAM1 expression which promotes antitumor immunity [20, 21]. Thus, interference in BRD7 expression offers therapeutic potential for inhibition of angiogenesis and tumor growth via multiple mechanisms.

\section{Materials and methods}

\section{Isolation and culture of endothelial cells}

EC were isolated from colorectal tumor tissues, patientmatched normal colon tissues and placenta tissues as previously described [3, 4]. Custom cDNA array screening [3] is detailed in the supplementary material and Fig. 1. HUVEC, HMEC-1 (referred to as HMEC, [22]) and EC-RF24 (referred to as RF24, [23]) were cultured as previously described [24, 25]. Where indicated, cells were treated for 3 days with $2 \mu \mathrm{M}$ sunitinib (Pfizer).

\section{Developmental CAM}

The CAMs on embryonic development day (EDD) 7 were divided into two treatment groups: control (topically $0.1 \%$ DMSO in $0.9 \% \mathrm{NaCl} ; 100 \mu \mathrm{l}$ injected) and sunitinib (topically $3.2 \mu \mathrm{g} / \mathrm{embryo} /$ day). The same treatment was repeated on EDD8. The CAMs were excised on EDD9 and processed for RNA isolation.

\section{Human tumors grown on the CAM}

Human ovarian carcinoma (A2780), human colorectal carcinoma (HCT116) and human breast carcinoma (MDAMB-231) (ECACC, Salisbury, UK) were maintained at $37{ }^{\circ} \mathrm{C}$ and $5 \% \mathrm{CO}_{2}$ in RPMI-1640 cell culture medium, supplemented with $10 \%$ FCS. A2780 and MDA-MB-231 tumors were implanted onto the CAM on EDD8 using hanging drops by preparing $25-\mu \mathrm{l}$ drops containing $1 \times 10^{6}$ cells, whereas for HCT116 $2.5 \times 10^{6}$ cells were applied in a 1:1 mix of medium and Matrigel (BD Biosciences). Where indicated, vascularized A2780 tumors were measured at EDD10, randomized and treated with vehicle (i.v. 0.1\% DMSO in $0.9 \% \mathrm{NaCl} ; 100 \mu \mathrm{l}$ injected) or sunitinib (i.v. $12 \mu \mathrm{g} / \mathrm{embryo} /$ day in $100 \mu \mathrm{l}$ vehicle). Xenograft tumors were excised on EDD15 and processed for RNA isolation.

\section{Quantitative real-time reverse transcription-PCR}

Total RNA was isolated using the RNeasy RNA isolation kit (Qiagen) and used for cDNA synthesis (Bio-Rad) according to the supplier's protocols. Cells were harvested by trypsinization and pelleted prior to cell lysis. Freshly resected colon tumor and adjacent normal colon tissue were minced and processed for RNA isolation. 
Fig. 1 Identification of genes downregulated in tumor EC. a Schematic overview of suppression subtractive hybridization (SSH) and differential (cDNA array) screening. Transcripts differing in abundance between tumor EC (TEC) and placenta EC (PLEC) or normal EC (NEC) are cloned in cDNA libraries. b The subtracted and enriched cloned repertoires were arrayed and probed with cDNA from TEC, NEC and PLEC. Shown are spot intensities in comparisons between TEC and NEC (left panel) and between TEC and PLEC (right panel). A clear bias toward genes suppressed in TEC is apparent, confirming successful subtraction. Spots showing $>3.5$-fold expression differences in either comparison (triangles) or in both comparisons (green diamonds) are indicated, as are the reference genes (blue crosses) and positive controls (red squares). c, d 170 spots showed $>3.5$-fold expression difference in TEC versus NEC and 216 spots showed $>3.5$-fold lower expression in TEC versus PLEC. 76 clones showed overlapping profiles (c) and were shown to represent 19 different mRNA transcripts (d). e Heatmap and unsupervised clustering on average Log 10 normalized intensities (see Table S1) of the top gene list
A

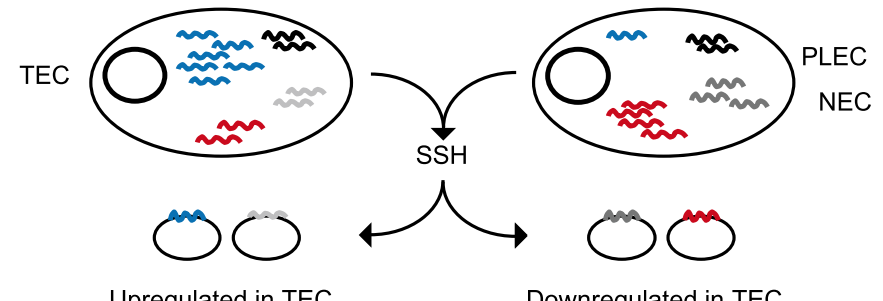

Upregulated in TEC

Downregulated in TEC
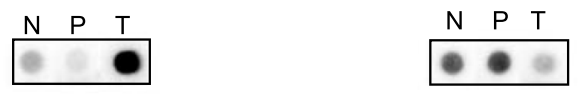

B
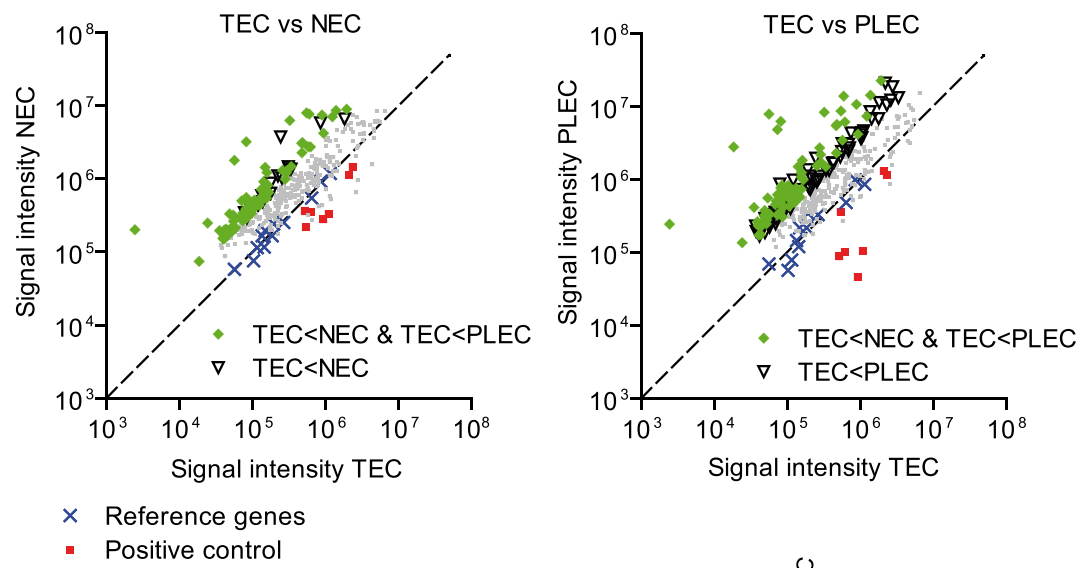

C

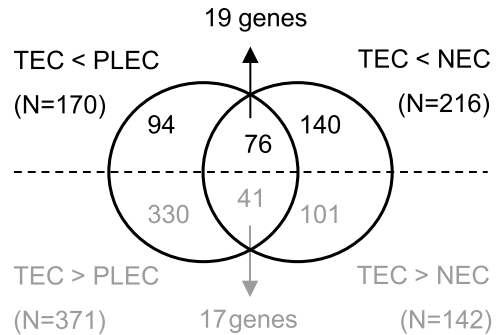

E
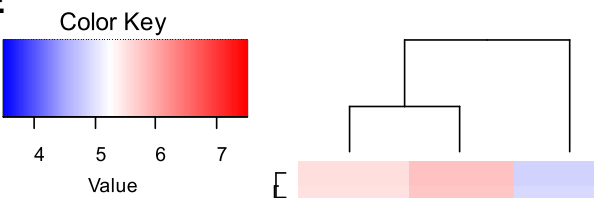

D 岩出

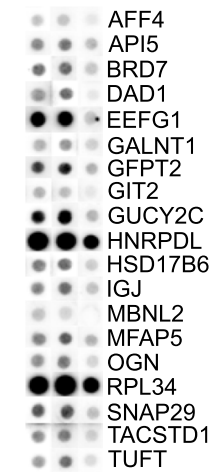

TUFT 
Primer design and validation was performed as described previously [26]. Primers specific for chicken BRD7, to selectively measure vascular BRD7 expression in the xenografted tumors, are presented in Fig. S1, and additional sequences are listed in Table S2. qPCR was performed using SYBR Green reagent (Bio-Rad). Assays were run on a CFX96 Real-Time PCR detection system and analyzed using BioRad CFX Manager software. Expression levels were defined using the $2^{\wedge}(-\mathrm{dCt})$ using mean expression of cyclophilin $\mathrm{A}$ (PPIA), beta-actin (ACTB) and beta-2-microglobulin (B2M) as reference genes. Where relevant, expression levels were expressed relative to one condition under study.

\section{BRD7 overexpression and knockdown}

Constructs containing Flag-tagged full-length (BRD7-FL) and bromodomain-deleted (aa129-237; BRD7-dBr) BRD7 open reading frames in pcDNA3 were a kind gift of Dr. Julia Kzyshkowska [27], and similar constructs in pEGFPN1 a kind gift of Dr. Jarno Drost [11]. For transfection, $1 \times 10^{5}$ RF24 cells were combined with 500 ng pDNA in resuspension buffer R (Life Technologies) in a 10- $\mu$ l tip and subject to 3 pulses of $10 \mathrm{~ms}$ at $1600 \mathrm{~V}$ in the Neon ${ }^{\circledR}$ Transfection system (Life Technologies). After electroporation, cells were seeded in 2 wells of 24-well plates to recover and used in downstream experiments after $48 \mathrm{~h}$. Controls represent cells transfected with the corresponding empty vectors.

Three different siRNAs targeting BRD7 were obtained from Qiagen (FlexiTube; SI04134088, SI04168059 and SI04320204), and control scrambled siRNA was obtained from Eurogentec. HUVEC were reverse transfected with $50 \mathrm{nM}$ siRNA as previously described [6].

\section{Functional assays}

EC proliferation was measured using a ${ }^{3} \mathrm{H}$-thymidine incorporation assay as described previously [3, 24]. For chemotaxis measurements, near-confluent HUVEC were starved overnight in medium containing $0.1 \%$ FCS prior to seeding $2.5 \times 10^{4}$ cells in a FluoroBlok insert with $8 \mu \mathrm{m}$ pore size (BD Biosciences) in a 24-well plate. The lower compartment of the well was filled with $200 \mu \mathrm{l}$ medium $(0.1 \%$ FCS $)$ combined with $50 \mu$ l conditioned medium of siRNA-transfected HUVEC. Plates were incubated overnight, and cells on the bottom side of the filter were stained with Calcein AM (Life Technologies). Five areas of the filters were photographed, and migrated cells were counted.

Active p65 NFкB subunit was detected using the EZdetect NFאB p65 ELISA assay kit (Pierce), according to the manufacturer's instructions. Briefly, cell lysates were incubated with p65 target DNA sequence immobilized in the assay plate. DNA-bound p65 was detected using antibodies in combination with chemiluminescence. Values were normalized to total protein content.

\section{Data mining and bioinformatics analysis}

Publicly available repositories were queried to obtain additional information on BRD7 gene and protein expression. The Protein Atlas (http://www.proteinatlas.org/) staining data were used to compare BRD7 protein expression in healthy and cancer tissues. NCBI GEO datasets GSE20076 [11] and GSE22607 [18] were analyzed to unravel the effects of BRD7 knockdown. GSE22607 contains expression data on BJ1 fibroblasts transduced with shBRD7 and GSE20076 contains expression data on RasV12 expressing BJ1 fibroblasts transduced with shBRD7. Briefly, using the GEO2R functionality, normalized $\log 2 \mathrm{FC}$ values of shBRD7-treated samples versus control samples were obtained and read into R. In addition, the subsets of genes showing differential expression after transfection of BRD7 in HEK293 fibroblasts reported by $\mathrm{Xu}$ et al. [28] were added as the full data set (GSE53656) was not publicly accessible. Data sets were merged and subsequently aggregated on gene names to have one unique identifier representing average expression of low versus high BRD7 expressing cells. FunRich (www.funrich. org), DAVID (https://david.ncifcrf.gov/), WebGestalt (www. webgestalt.org) and PathVisio (www.pathvisio.org) were used to perform functional annotation analysis on the final data set.

\section{Statistical analyses}

All values are given as mean values \pm SEM. Statistical analyses were done using either $t$ test, Mann-Whitney $U$ (M-W) or Wilcoxon rank sum test (Wilcoxon) for single comparisons, or, where appropriate, one-way ANOVA or Kruskal-Wallis (K-W) in combination with Dunnett's multiple test correction. All analyses were done in GraphPad Prism 3.0. $p$ values $<0.05$ were considered statistically significant.

\section{Results}

\section{BRD7 expression is inhibited in tumor endothelium}

Gene expression profiling of freshly isolated endothelial cells (EC) from colon tumors, normal colon and placenta identified 19 genes that were specifically suppressed in tumor EC (TEC) (Fig. 1; Table S1). The reported downregulation of BRD7 in cancer [13, 14, 29] prompted us to further elucidate the role of BRD7 in tumor angiogenesis. qPCR validated the differential BRD7 expression in isolated EC. Not only is BRD7 mRNA specifically 
downregulated in TEC (Fig. 2a), global BRD7 mRNA expression was reduced in a panel of colorectal tumors compared to normal colon (Fig. 2b), confirming previous reports [30]. BRD7 protein in normal colon tissue sections was clearly associated with the vasculature (Fig. 2c i, ii), both in the endothelial cell layer and in underlying vascular structures such as the vascular smooth muscle layer. Vascular BRD7 expression was virtually absent in colon tumor sections (Fig. 2c iii, iv). In addition, mining The Protein Atlas data also revealed a reduction in BRD7 protein expression in colon tumors (Fig. 2d) as compared to normal colon.
While in tumor cells loss of chromosome 16q12 may be responsible for reduced expression of BRD7 [31], loss of heterozygosity is not a common phenomenon in TEC which are considered non-transformed cells [32]. Epigenetic silencing of BRD7 expression through promoter $\mathrm{CpG}$ island methylation and/or histone deacetylation in EC would be feasible [9, 33], but was not observed (Fig. S2).

Suppression of tumor EC BRD7 expression appears to be a generic feature in cancer. Using PCR primers specific for chicken BRD7 (Fig. S1), we profiled the vascular BRD7 expression in different human tumors (colon, breast and
A

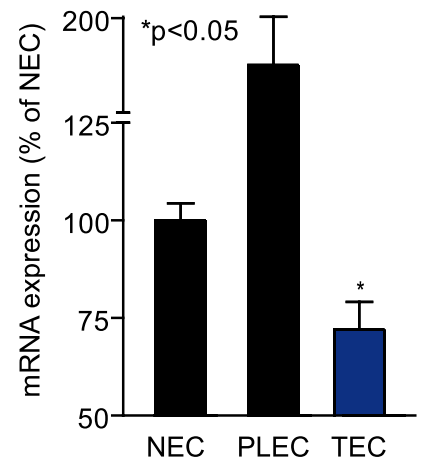

E

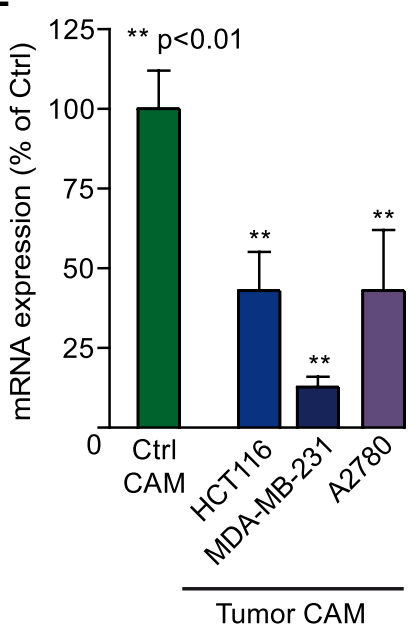

B

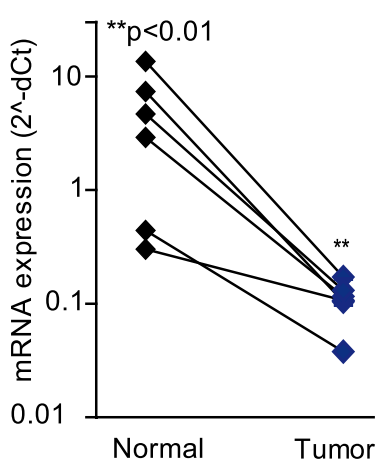

D

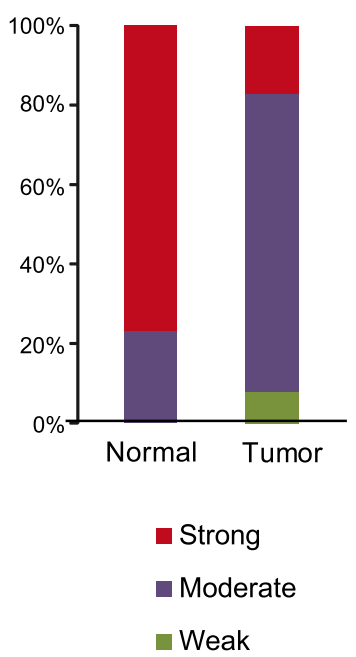

C
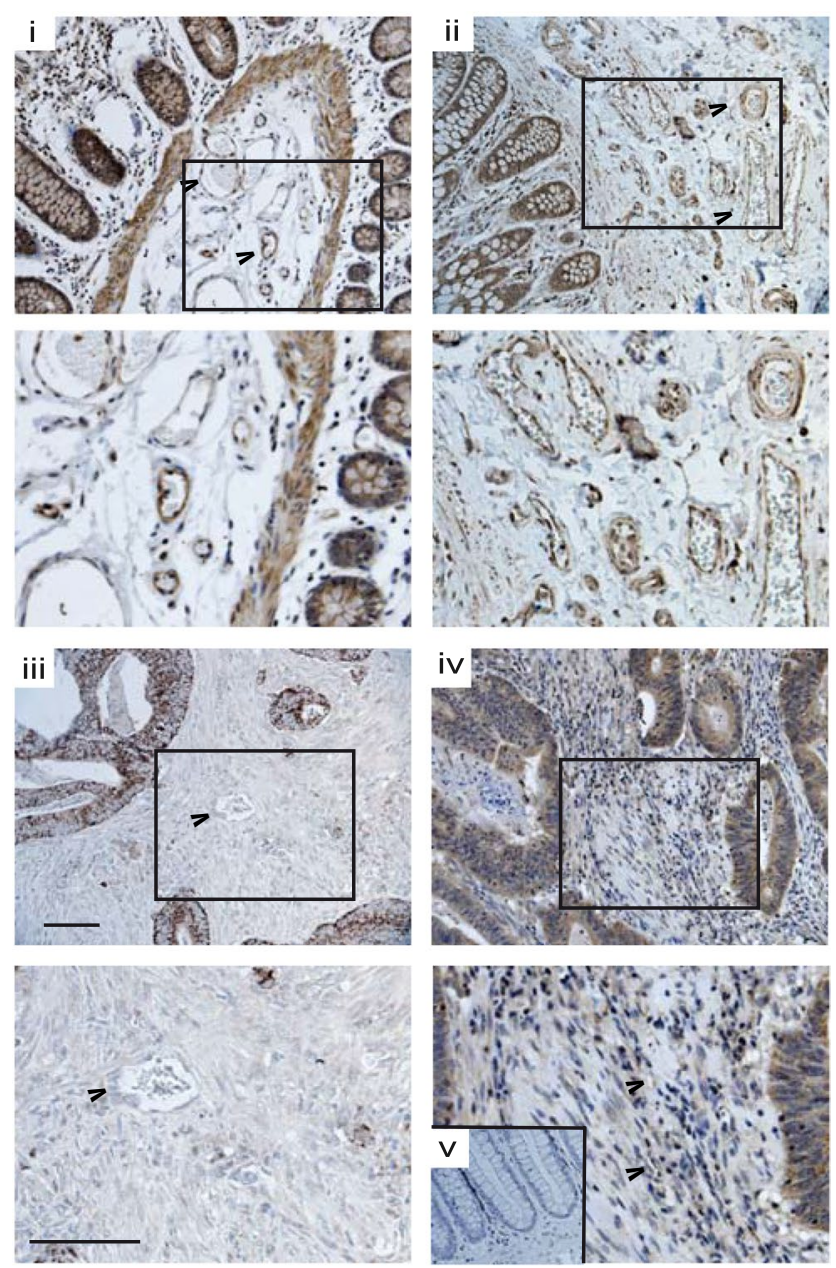

respective panels below. For indicative purposes, several blood vessels are indicated with arrowheads. Scale bar $=100 \mu \mathrm{m}$. d Distribution of staining intensities in colon tumors and normal colon samples $(N=12)$ reported in The Protein Atlas indicates less intense staining in tumor tissues. e BRD7 mRNA expression is reduced in the (chicken derived) vasculature of different human tumors (colon, breast and ovary) xenografted on the CAM (Tumor CAM) in comparison with developmental CAM (Ctrl CAM), as determined by qPCR using chicken-specific primers. ${ }^{*} p<0.01$ ANOVA. All data are presented as mean \pm SEM 
ovary) xenografted on the chicken chorioallantoic membrane (CAM) [34-36]. Figure 2e clearly shows suppressed BRD7 expression in the tumor blood vessels on the CAM as compared to normal CAM vessels (Ctrl CAM). Thus, in addition to its tumor suppressive properties, the expression profile of BRD7 is also suggestive of angiosuppressor functions.

\section{BRD7 expression is negatively associated with endothelial cell activation}

To determine how BRD7 expression is regulated in EC, freshly isolated quiescent human umbilical vein EC (HUVEC) (P0) were compared with cultured, serum-activated HUVEC (P1), demonstrating a clear suppression of BRD7 transcript upon propagation in vitro (Fig. 3a). Restoration of original BRD7 expression levels was accomplished by serum withdrawal (S) (Fig. 3a). Serum depletion of HMEC and RF24 also resulted in enhanced BRD7 expression, comparable to that seen for HUVEC (Fig. S3). In addition, when the expression levels of BRD7 in the immortalized, fast-growing EC lines HMEC and RF24 were compared with that of primary HUVEC, a markedly higher expression was observed in primary, slower-growing HUVEC, further suggesting an inverse association between BRD7 transcript levels and EC proliferation rates (Fig. 3b). Thus, expression of BRD7 appears to be influenced by microenvironmental stimuli, as well as by endogenous growth rates of the EC.

The clear relationship between BRD7 expression levels and EC growth prompted us to investigate whether BRD7 expression is altered upon treatment of EC with angiogenesis inhibitors. EC were treated with sunitinib, and BRD7 expression was monitored by qPCR. The receptor tyrosine kinase inhibitor sunitinib, but not the anti-VEGF antibody bevacizumab (data not shown), induced the expression of BRD7 (Fig. 3c, d), which corresponds to the effective inhibition of EC growth in vitro by sunitinib though not bevacizumab [36, 37]. A similar expression regulation of BRD7 was observed in the developmental CAM in vivo (Fig. 2e). Xenograft tumors treated with sunitinib showed enhanced expression of endothelial BRD7 (Fig. 3f) and displayed inhibited growth [35]. Together, these data further support the notion that BRD7 expression levels are negatively associated with EC growth.

\section{BRD7 exerts an angiosuppressive function}

To functionally evaluate the role of BRD7 and specifically its bromodomain in EC biology, RF24 cells were transfected with previously validated expression constructs encoding either full-length BRD7 protein (BRD7-FL) or BRD7 protein with deleted bromodomain (BRD7-dBr) [11, 27], resulting in a clear induction of BRD7 expression (Fig. 4a).
Transfection efficiencies were at least 50\%, comparable for the different constructs, consequent for mRNA and protein induction, and displayed only moderate impairment of cell viability. In addition, we observed no difference in expression of BRD7 in empty vector-transfected cells as compared to untransfected cells, essentially ruling out artifacts related to the procedure (Fig S4 and data not shown). We next investigated the cell growth properties of EC transfected with the different BRD7 expression constructs. As is evident from Fig. 4b, introduction of BRD7 resulted in a drastic inhibition of EC proliferation, which was partially overcome by deletion of the bromodomain. Comparable results were obtained with GFP-tagged and Flag-tagged BRD7 expression constructs. Moreover, using GFP-tagged expression constructs, which allowed direct monitoring of successfully transfected cells, we observed that the majority BRD7 expressing GFP-positive cells failed to adhere to the growth substrate indicating severe loss of viability and adhesive properties upon BRD7 expression (Fig. 4c). Since five to tenfold overexpression resulted in considerable suppression of EC proliferation, we considered these cells too impaired for further assessment of sprouting capacity.

Using siRNA to knock down BRD7 expression, we sought to reverse the phenotypic effects observed with the expression constructs. BRD7 expression was profoundly suppressed (Fig. 4d). However, we did not observe effects on EC proliferation (Figs. 4e, S4) and scratch wound migration (data not shown). Comparable results were obtained with two of the three independent BRD7-specific siRNAs (Fig $\mathrm{S} 4$ and data not shown). All data were expressed relative to a scrambled siRNA control as to exclude off-target effects.

The lack of phenotype may be related to the intrinsically high activation status of cultured EC in vitro, which leaves a too narrow detection window for additional activation as a consequence of BRD7 suppression. Of note, we selected HUVEC for these experiments as they express the highest levels of BRD7 and display the lowest level of proliferation when compared to HMEC and RF24 (Fig. 3b). Furthermore, serum starvation of the cells after the transfection procedure did not induce any divergent responses in siBRD7- versus siCtrl-transfected cells. However, chemotactic migration of naïve cells toward conditioned medium of siBRD7-treated cells was enhanced (Fig. 4f) and appeared to be associated with more intense Calcein AM fluorescence (Fig. 4f, right panel), suggestive of increased viability. Nevertheless, quantification of fluorescence intensity did not reveal a significant increase (data not shown).

\section{BRD7 affects inflammatory and angiogenic cytokine expression}

To further elucidate the mechanism by which BRD7 affects EC activation, we profiled a panel of angiogenic factors and 
A

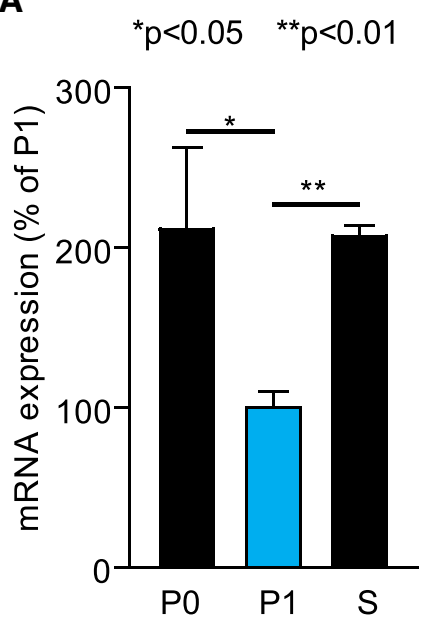

B

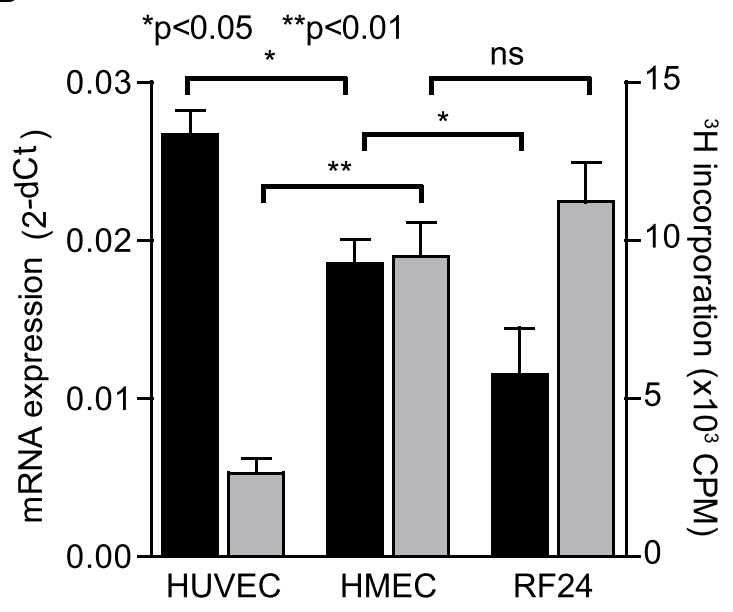

C

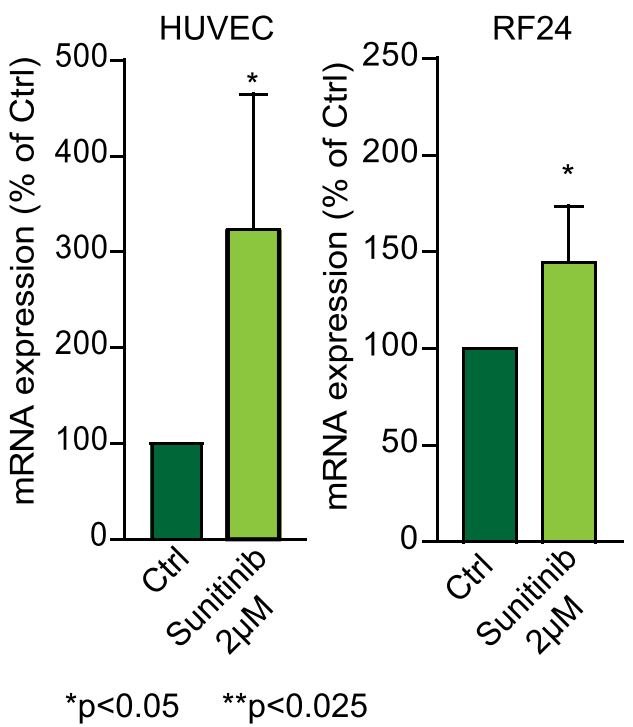

$\mathbf{E}$

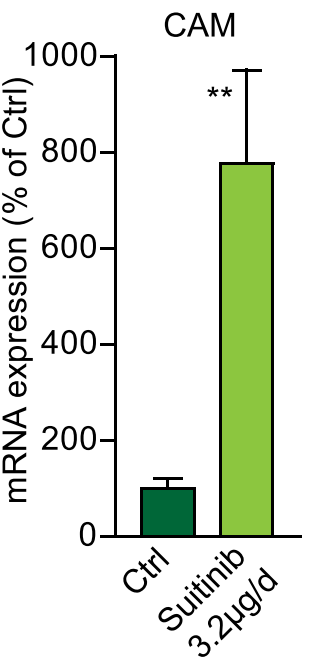

$\mathbf{F}$

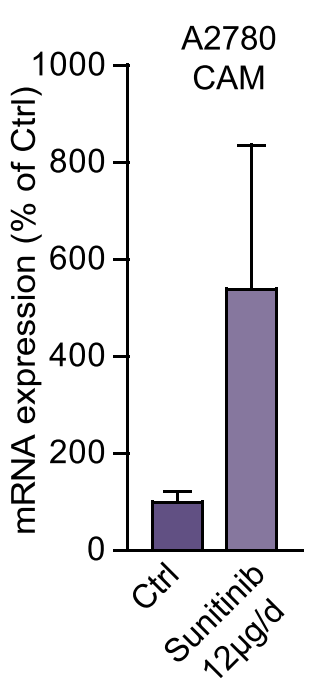

Fig. 3 BRD7 expression is inversely related to endothelial cell growth. a HUVEC were isolated as described and either processed for RNA isolation immediately after isolation (P0) or grown under standard conditions for 3 days (P1), and BRD7 transcript levels were measured by qPCR. A clear reduction of BRD7 mRNA is evident in cells stimulated to proliferate in vitro. Serum withdrawal (S) of established HUVEC increases the BRD7 mRNA levels to that of primary isolates. ${ }^{*} p<0.05,{ }^{*} p<0.01 t$ test. b BRD7 expression was measured in routinely cultured HUVEC, HMEC and RF24 by qPCR. In parallel, proliferation rate of the cells was measured by ${ }^{3} \mathrm{H}$-thymidine incorporation. Primary cells (HUVEC) show higher expression levels (black bars; left $y$-axis) than immortalized EC (RF24 and

their receptors in BRD7-transfected (BRD7-FL and BRD7$\mathrm{dBr}$ ) or empty vector-transfected EC (Ctrl) by qPCR. From Fig. S4a, it is clear that overexpression of BRD7-FL or $\mathrm{BRD} 7-\mathrm{dBr}$ does not have a major influence on the expression of angiogenic growth factors and their receptors involved in signaling along the VEGF/VEGFR or angiopoietin/Tie axis.
HMEC), but the opposite holds true for proliferation rate (gray bars; right $y$-axis). $* p<0.05$ Wilcoxon test. c, d HUVEC (c) and RF24 (d) were treated with $2 \mu \mathrm{M}$ sunitinib and BRD7 was quantified by qPCR. Sunitinib markedly increases BRD7 expression. $* p<0.05$ Wilcoxon test. e CAMs were treated with $3.2 \mu \mathrm{g}$ sunitinib per day, and chicken BRD7 expression was determined with qPCR. Similar to in vitro treatment, in vivo treatment with sunitinib increases BRD7 expression. $* * p<0.01$ ANOVA. f sunitinib treatment (12 $\mu \mathrm{g} /$ day) of A2780 tumors on the CAM results in an increase in vascular BRD7 expression as determined by qPCR. $* p=0.05$ Mann-Whitney test. All data are presented as mean \pm SEM

Moderate changes were observed with BRD7 knockdown, most notable the upregulation of VEGF receptor-1 (FLT1), angiopoietin-2 (ANGPT2) and neuropilin-1 (NRP1) (Fig. $\mathrm{S} 4 \mathrm{~b}, \mathrm{c})$. In contrast, TNF- $\alpha$ was markedly upregulated after overexpression of full-length BRD7 but not after overexpression of the bromodomain-deficient protein (Fig. 5a), and this 
A

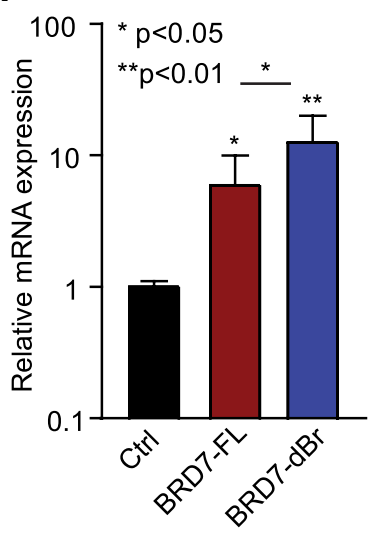

B

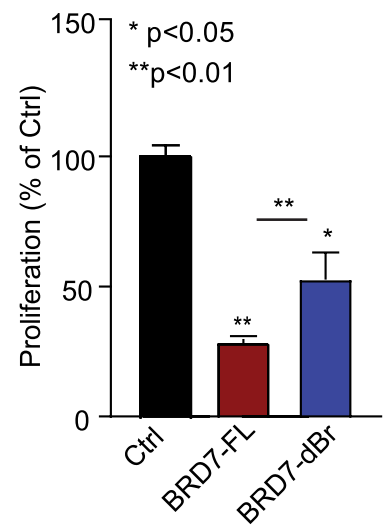

E
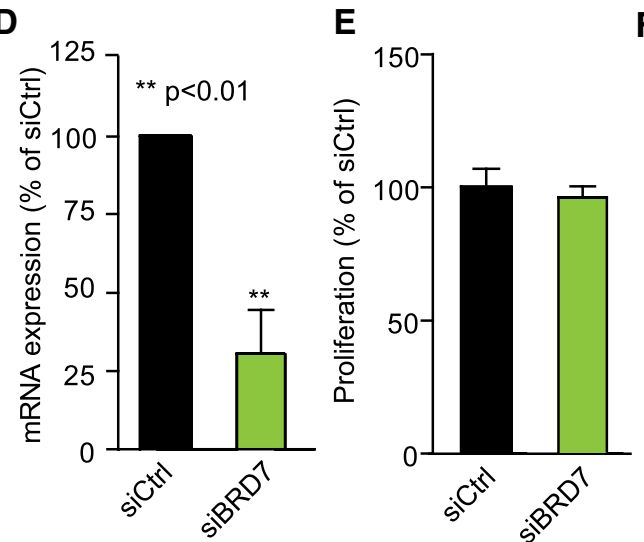

Fig. 4 Functional effects of BRD7 overexpression and knockdown in endothelial cells. a, b Full-length BRD7 (BRD7-FL) and bromodomain deletion mutant (BRD7-dBr) cloned in pcDNA3 [27] were ectopically expressed in RF24. Control cells were transfected with empty pcDNA3. This resulted in seven to tenfold increased in BRD7 expression (a). BRD7 overexpression results in inhibition of EC proliferation as measured by ${ }^{3} \mathrm{H}$-thymidine incorporation assay (b). c Overexpression of BRD7 and BRD7-dBr results in a reduced frac-
F

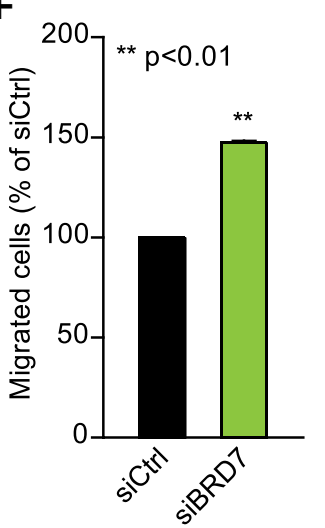

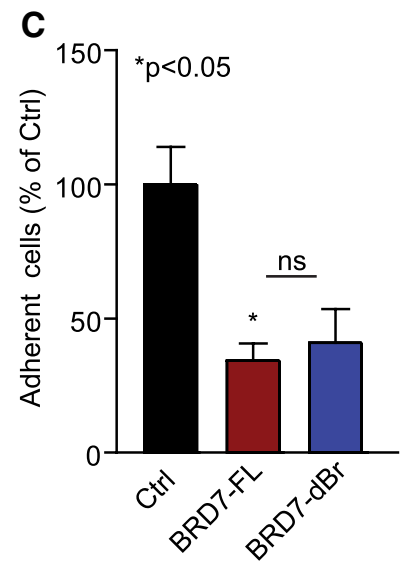
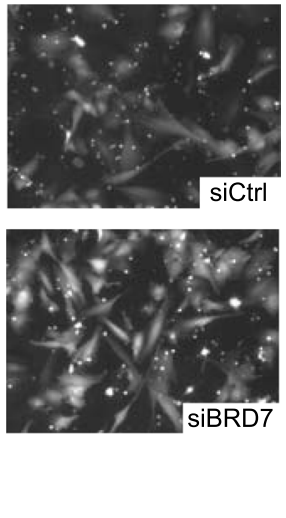

tion of adherent cells. Cells were transfected with GFP-tagged BRD7 expression constructs or empty pEGFPN1 vector, which allowed easy identification of successfully transfected cells. $* p<0.05,{ }^{*} p<0.01$ ANOVA. d-f Knockdown of BRD7 by siRNA in HUVEC (d) does not affect proliferation of cells (e). Migration toward conditioned medium of siBRD7-treated cells was enhanced (f). $* * p<0.01 t$ test. All data are presented as mean \pm SEM

gene ontologies using DAVID, however, showed several similarities. The fraction of genes associated with low BRD7 expression displayed enrichment for genes associated with inflammatory responses and cytokine activity, as well as for angiogenesis (Table S5). A similar profile was apparent for ontology analysis of the merged data set (Fig. 6b, c).

Cytokines CXCL1 and CXCL6 were differentially regulated in all three expression datasets under investigation and were confirmed to be upregulated after BRD7 knockdown and somewhat suppressed after overexpression EC (Fig. 5c, e). In addition, NOTCH1, previously identified as a transcriptional target of BRD7 [28], was subject to BRD7induced regulation (Fig. 5c, e).

Using PathVisio [39] and the merged expression data set containing average $\log 2 \mathrm{FC}$ values, we constructed a schematic representation on BRD7 angiosuppressive actions with expression changes color-coded (Fig. 6a). 
A

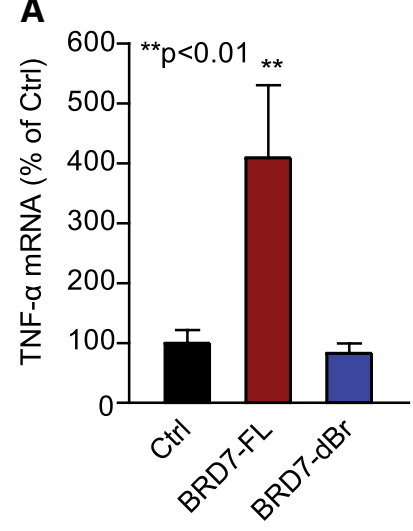

D

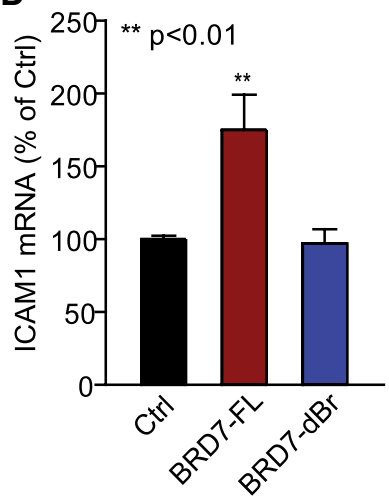

$\mathbf{F}$

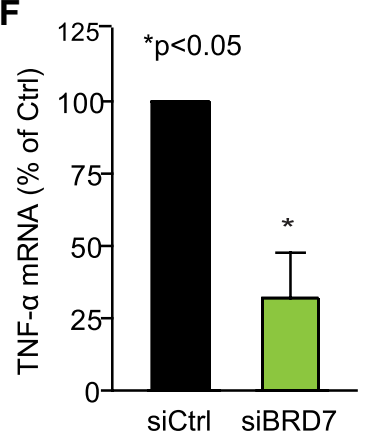

B
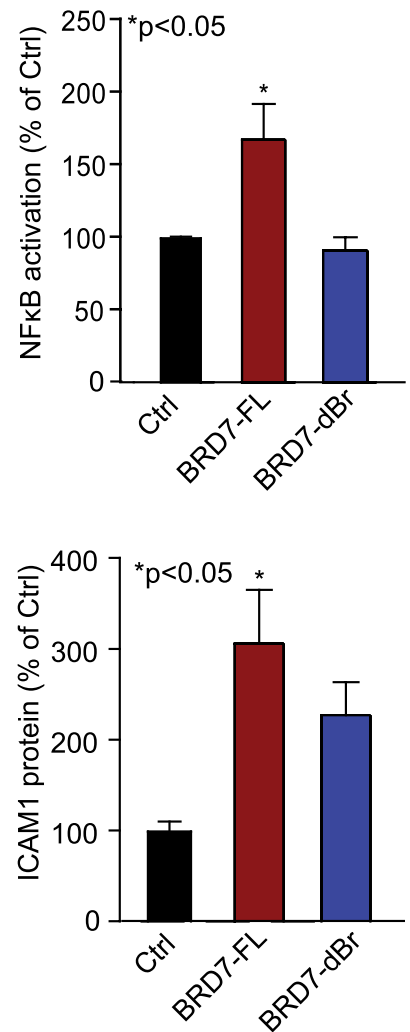

G

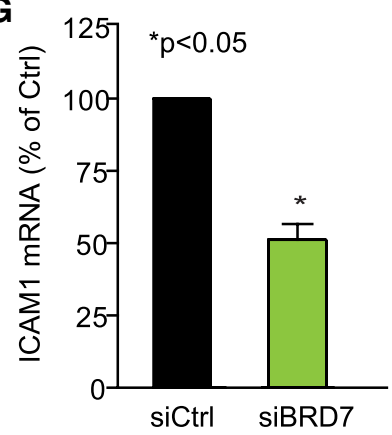

C

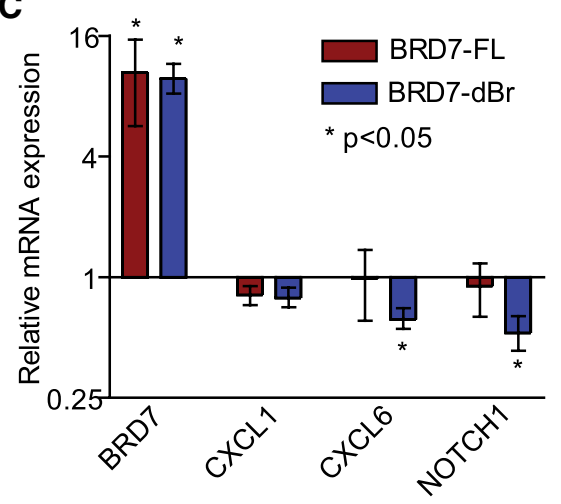

E
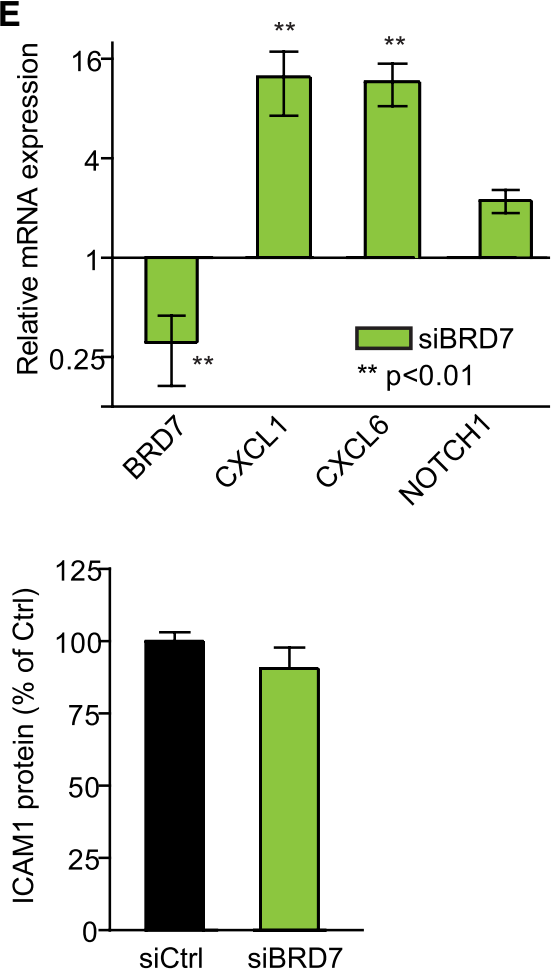

Fig. 5 BRD7 expression modulation affects angiogenic and inflammatory gene expression. a-d Full-length BRD7 (BRD7-FL) and bromodomain deletion mutant (BRD7-dBr) were ectopically expressed in RF24, which resulted in a bromodomain-dependent increase in TNF- $\alpha$ expression (a), NFkB activation (b), and ICAM1 mRNA and protein (d). Expression of CXCL1, CXCL6 and NOTCH1 is only slightly affected (c). e- $\mathbf{g}$ mRNA expression changes are reversed upon knockdown of BRD7, although ICAM1 protein expression was not affected. $* p<0.05, * * p<0.01$ ANOVA. All data are presented as mean \pm SEM
Downregulation of BRD7 was accompanied by NOTCH1, CXCL1 and CXCL6 upregulation and NFKB downregulation, which we experimentally confirmed (Fig. 5). Using overrepresentation analysis, pathways and ontologies associated with cytokine signaling, chemotaxis and migration, as well as NFKB and TNF- $\alpha$ signaling were shown to be enriched in cells with suppressed BRD7 expression (Fig. 6b, c). Together, these data point to a multifaceted action of BRD7 in regulating the expression of different angiogenic mediators.

\section{Discussion}

In this report, we describe the identification of BRD7 as a negative regulator of angiogenesis. BRD7 expression was found to be suppressed in endothelial cells (EC) isolated from human colon tumors as compared to isolated EC from normal colon tissue. BRD7 has been described as a putative tumor suppressor protein, which prompted us to further investigate its role in tumor angiogenesis. 


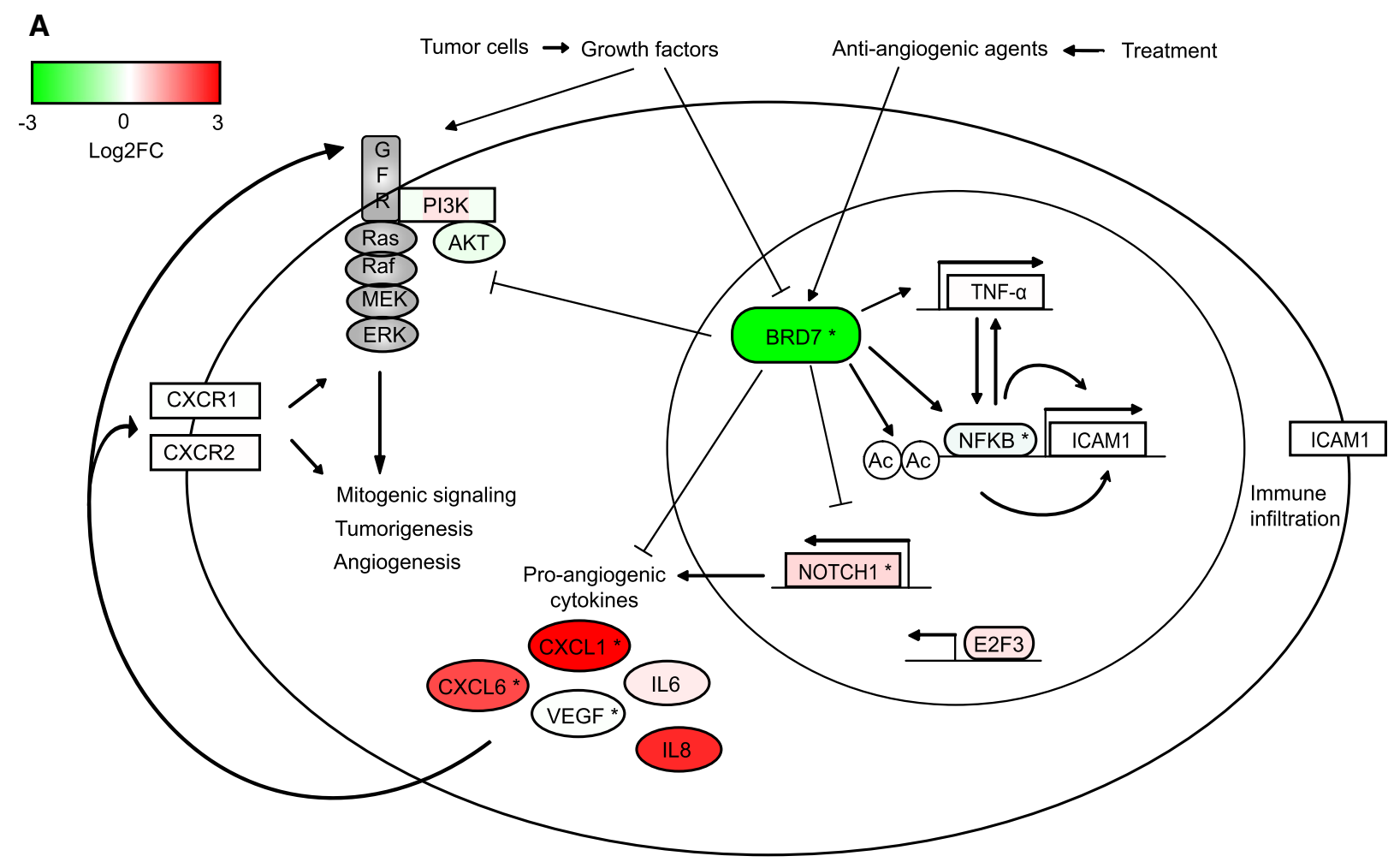

B

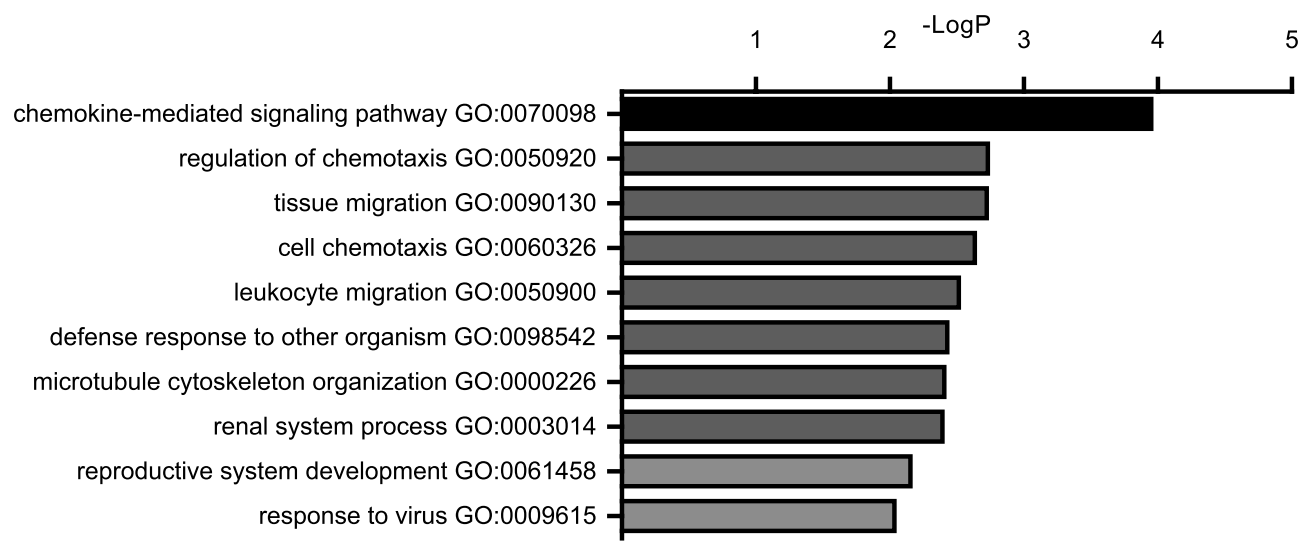

C

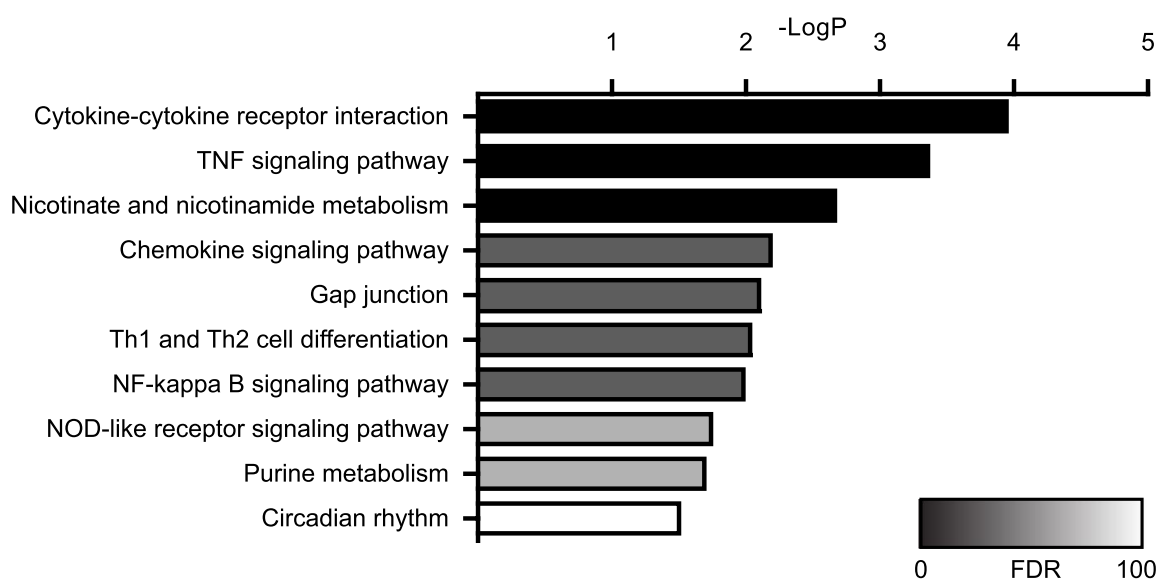


\Fig. 6 Schematic representation of the multifaceted angiosuppressor function of BRD7. a BRD7 expression is stimulated by angiogenesis inhibitors and repressed by growth factor exposure, such as those produced by tumor cells. BRD7 inhibits mitogenic Ras-RafMEK-ERK and PI3K-Akt signaling, as well as E2F3 transcription factor activity and NOTCH1 expression that also normally result in enhanced cell growth and contribute to angiogenesis and tumorigenesis. Low BRD7 levels are related to enhanced CXCL1, CXCL6, IL6 and IL8 cytokine production, which can also promote tumor growth and angiogenesis. On the other hand, BRD7 can directly or indirectly increase TNF- $\alpha$ expression and stimulates NFKB activation, resulting in increased expression of downstream target genes including ICAM1. Furthermore, BRD7 might also directly facilitate ICAM1 transcription by interaction with acetylated histones in the ICAM1 promoter sequence. Upregulation of ICAM1 results in increased EC-leukocyte interactions and positively contributes to immune cell infiltration into tumors. Colors reflect average expression changes in the analyzed data sets. * indicates concordant regulation in endothelial cells reported in this work. b, $\mathbf{c}$ Overrepresentation analysis of gene ontologies (B) or KEGG pathways (c) in differentially expressed $(\log 2 \mathrm{FC}>2)$ genes $(N=290)$ after BRD7 knockdown using WebGestalt tool. Top 10 categories are shown with $p$ values. Bars are color-coded for false discovery rate (FDR). Of note, no significantly enriched ontologies were found with $\log 2 \mathrm{FC}<2$ genes following BRD7 knockdown $(N=360)$ (data not shown)

We confirmed suppressed BRD7 expression both in isolated EC and reduction of BRD7 expression in tumor endothelium was independently observed in different xenografted tumors on the CAM, suggesting a generic cancer feature. Unfortunately, no relevant public data sets to independently validate BRD7 expression in human isolated TEC are available. The sparse availability of tissue-isolated EC precluded us also from quantifying BRD7 protein levels in these cells separately. However, immunohistochemistry of colorectal cancer and normal tissues confirms the downregulation of BRD7 protein in the tumor vasculature. In concordance with these findings, BRD7 expression levels were inversely related with EC activation status in vitro. In addition, treatment with the anti-angiogenic tyrosine kinase inhibitor (TKI) sunitinib increased BRD7 expression levels in vitro and in vivo. This relatively broad spectrum TKI may affect multiple phosphorylation pathways that (in)directly affect the regulation of BRD7 expression.

Ectopic expression of BRD7 resulted in decreased proliferation of EC, and this phenotype was partially dependent on the presence of the bromodomain. So far, deletion of the bromodomain in BRD7 has been associated with a reduced binding capacity to acetylated histones [27], and a loss of inhibition of E2F3 promoter activity [17, 29]. Also, nuclear expression of BRD7 lacking bromodomain is more granular [17], likely caused by an altered ability to bind chromatin. However, the precise mode of action of BRD7, and more specifically the role of its bromodomain in regulating cell growth has not been fully elucidated. Our data indicate that the bromodomain of BRD7 is involved in regulating NFKB activation and the expression of downstream molecules
(TNF- $\alpha$, ICAM1), but not that of other molecules such as CXCL1, CXCL6 and NOTCH1.

In previous studies $[8,9]$, we profiled epigenetic silencing in endothelial cells and demonstrated a pivotal role for ICAM1. Endothelial ICAM1 is one of the key adhesion molecules that mediate leukocyte rolling and adhesion, and hence contributes to leukocyte infiltration and anti-tumor immunity. In tumors, however, the expression of ICAM1 on EC is suppressed, and thus, tumors may escape from immune surveillance $[8,20,21]$. As we have demonstrated in the past, anti-angiogenic treatment can overcome the downregulation of ICAM1 and restore leukocyte infiltration [20], and as shown here, this is accompanied by, and may be a direct consequence of, an increase in BRD7 expression. ICAM1 can be regulated via TNF- $\alpha$-induced NFKB-dependent transcription, but TNF- $\alpha$ expression itself can also be controlled by NFkB, creating a positive feedback loop [21]. Binding of NFKB p65 subunit to its target sequence was enhanced in cells overexpressing BRD7-FL, but not BRD7-dBr, suggesting BRD7 stimulates NFKB-dependent transcription in a bromodomain-dependent fashion. Though we did not investigate direct binding of BRD7 to NFKB, in this context, it is highly interesting to note that recently the related BRD4 protein was shown to bind to RelA and to activate NFKB by a bromodomain-dependent mechanism [40]. Alternatively, BRD7 could, through its bromodomain, bind acetylated histones that are present in the ICAM1 promoter and positively regulate its expression [8]. A recent chromatin-IP study did, however, not identify these genes as direct transcriptional targets of BRD7 [28]. Nevertheless, BRD7 is known to act in larger complexes and interactions may be cell-type dependent $[11,15,18]$.

$\mathrm{NFkB}$ plays a paradoxical role in tumor growth and tumor angiogenesis. In tumor cells, its activation is altered and it drives the production of pro-angiogenic cytokines, including CXCL1 and CXCL6. In addition, it feeds a positive prometastatic and pro-angiogenic feedback loop [41] and inhibits pro-apoptotic genes [38]. However, in EC NFKB plays a divergent role, as it may play a predominant pro-apoptotic role, induces the expression of anti-angiogenic factors and is involved in effectuating angiostatic therapy and increasing anti-tumor immunity [38]. In addition, in HUVEC, but not in fibroblasts, it plays a pivotal role in senescence induction [42, 43], whereas blockade of NFKB could overcome aging [44]. Interestingly, recently XAF1, a transcriptional target of BRD7 which acts analogously to NFKB in inhibiting XIAP, was shown to play a role in endothelial senescence [19].

The data presented in this study also point to a unique positioning of $\mathrm{NFKB}$ in $\mathrm{EC}$. While studies in fibroblasts employing BRD7 expression modulation report induction of the pro-angiogenic cytokines CXCL1 and CXCL6 upon BRD7 silencing, upregulation of ICAM1 and NFKB are concomitantly observed [11, 18, 28, 45]. In contrast, in EC 
BRD7 acts in an opposite manner on NFkB and ICAM1. To further unravel the differential actions of BRD7 in relation to NFkB-mediated processes, as well as its in vivo contribution of tumor angiogenesis, the generation of genetically engineered models of (conditional) vascular BRD7 knockout, combined with ChIP-seq analysis, would be highly valuable.

BRD7 has been described as a subunit of the SWI/SNF chromatin remodeling complex PBAF [16]. Involvement of this complex in angiogenesis was shown by knockdown of the accompanying BRG1 subunit in embryonic stem cells, which resulted in the induction of angiogenesis associated genes [16]. However, a later study employing vascular specific knockout of BRG1 yielded no observable effects on postnatal angiogenesis [46]. While BRG1 can participate in both BAF and PBAF, BRD7 is specific for PBAF [16]. As such, depletion of BRG1 implies a functional deletion of BRD7 action within this complex. Indeed, transcriptional changes upon BRG1 knockdown mimicked those of BRD7 suppression such as shown here [16, 47, 48]. The apparent absence of phenotype upon knockdown is in line with our observations that suppression of BRD7 expression has less effects than overexpression, suggesting that compensatory mechanisms likely play a role.

Endothelial BRD7 contributes to suppression of tumor angiogenesis in a number of ways, schematically visualized in Fig. 6 and reconstructed from data presented here combined with published data. BRD7 inhibits mitogenic signaling, tumorigenesis and angiogenesis and stimulates anti-tumor immunity through different possible pathways. BRD7 negatively regulates the Ras-Raf-MEK-ERK mitogenic and PI3K-Akt signaling cascades [17, 28, 29, 49], the latter presumably through direct binding of PI3K subunit p85 $\alpha$ [49]. Furthermore, BRD7 inhibits E2F3 [17, 29, 50] and NOTCH1 [28] promoter activity. Interestingly, blockade of the NOTCH signaling pathway has been employed to target tumor angiogenesis, although the complex interplay of its different ligands complicate predicting the outcome of such strategies [51]. The discovery that knockdown of BRD7 results in induction of pro-angiogenic cytokines, most concordantly CXCL1 and CXCL6 expression (Figs. 5, 6, S5; Tables S3, S4), adds an additional layer to its angiomodulatory actions. CXCL1, previously known as GRO1 oncogene, has mitogenic, angiogenic and tumorigenic properties [52]. CXCL6, also known as GCP-2, is another pro-angiogenic cytokine with tumor growth promoting properties [53], and anti-CXCL6 antibodies reduced tumor growth and metastasis formation in vivo [54]. Moreover, CXCL6 can stimulate the production of other pro-angiogenic factors such as VEGFA, HGF and IL8 [55]. Interestingly, the previously reported tumor angiogenesis gene $\operatorname{HMGB} 1[3,6,56]$ displays parallel actions to BRD7 knockdown as it induces the expression of different pro-angiogenic cytokines, including IL8, CXCL1 and CXCL6 [57]. This might feed an autocrine and/or paracrine positive feedback mechanism [56], where tumor cells and EC can reciprocally stimulate each other's growth [58], contributing to tumor progression and sustained angiogenic activation [17, 28, 29, 49].

In summary, we identified a novel angiosuppressor gene that extends our understanding of the physiology of tumor EC and provides multiple novel opportunities for interfering with tumor angiogenesis. Therapeutic induction of BRD7 expression might not only be valuable in reducing tumor cell proliferation, but also in reducing tumor angiogenesis and increasing anti-tumor immune infiltration. This proposed triple-targeting of cancer progression offers a unique and new approach in the battle against cancer, and may prove superior to monotherapies.

Acknowledgements This work was supported by the sixth EU Framework Programme (Integrated Project 'Angiotargeting'; Contract No. 504743) in the area of 'Life sciences, genomics and biotechnology for health,' the School for Life Sciences, transnational University Limburg (tUL), Maastricht, CTMM Mammoth and KWF (VU20125715). This study was further supported by the European Union (PIEFGA-2013-626797 and ERC-2015-StG-LS7-680209) to PNS. We thank Wouter Eijgelaar, Dannielle Verboogen, Edith van der Linden, Veerle Melotte and Petra Hautvast (MUMC) for expert technical assistance.

Author contributions JRvB and AWG conceived experiments. JRvB, PNS, MvB and TW carried out experiments. JRvB and PNS analyzed data. $J R v B$ wrote the manuscript.

\section{Compliance with ethical standards}

Conflict of interest The authors declare no conflicts of interest.

Open Access This article is distributed under the terms of the Creative Commons Attribution 4.0 International License (http://creativecommons.org/licenses/by/4.0/), which permits unrestricted use, distribution, and reproduction in any medium, provided you give appropriate credit to the original author(s) and the source, provide a link to the Creative Commons license, and indicate if changes were made.

\section{References}

1. Hanahan D, Weinberg RAA (2011) Hallmarks of cancer: the next generation. Cell 144:646-674. doi:10.1016/j.cell.2011.02.013

2. van Beijnum JR, Nowak-Sliwinska P, Huijbers EJM et al (2015) The great escape; the hallmarks of resistance to antiangiogenic therapy. Pharmacol Rev 67:441-461. doi:10.1124/pr.114.010215

3. van Beijnum JR, Dings RP, van der Linden E et al (2006) Gene expression of tumor angiogenesis dissected: specific targeting of colon cancer angiogenic vasculature. Blood 108:2339-2348. doi:10.1182/blood-2006-02-004291

4. van Beijnum JR, Rousch M, Castermans K et al (2008) Isolation of endothelial cells from fresh tissues. Nat Protoc 3:1085-1091. doi:10.1038/nprot.2008.71

5. St Croix B, Rago C, Velculescu V et al (2000) Genes expressed in human tumor endothelium. Science 289:1197-1202. doi:10.1126/ science.289.5482.1197

6. van Beijnum JR, Nowak-Sliwinska P, van den Boezem E et al (2013) Tumor angiogenesis is enforced by autocrine 
regulation of high-mobility group box 1 . Oncogene 32:363-374. doi:10.1038/onc.2012.49

7. Thijssen VLJL, Postel R, Brandwijk RJMGE et al (2006) Galectin-1 is essential in tumor angiogenesis and is a target for antiangiogenesis therapy. Proc Natl Acad Sci USA 103:15975-15980. doi:10.1073/pnas.0603883103

8. Hellebrekers DMEI, Castermans K, Viré E et al (2006) Epigenetic regulation of tumor endothelial cell anergy: silencing of intercellular adhesion molecule- 1 by histone modifications. Cancer Res 66:10770-10777. doi:10.1158/0008-5472. CAN-06-1609

9. Hellebrekers DMEI, Melotte V, Viré E et al (2007) Identification of epigenetically silenced genes in tumor endothelial cells. Cancer Res 67:4138-4148. doi:10.1158/0008-5472.CAN-06-3032

10. Luo W, Hu Q, Wang D et al (2013) Isolation and genome-wide expression and methylation characterization of $\mathrm{CD} 31+$ cells from normal and malignant human prostate tissue. Oncotarget 4:14721483. doi: $10.18632 /$ oncotarget. 1269

11. Drost J, Mantovani F, Tocco F et al (2010) BRD7 is a candidate tumour suppressor gene required for $\mathrm{p} 53$ function. Nat Cell Biol 12:380-389. doi:10.1038/ncb2038

12. Mantovani F, Drost J, Voorhoeve PM et al (2010) Gene regulation and tumor suppression by the bromodomain-containing protein BRD7. Cell Cycle 9:2777-2781. doi:10.4161/cc.9.14.12309

13. Park YA, Lee JW, Kim HS et al (2014) Tumor suppressive effects of bromodomain-containing protein 7 (BRD7) in epithelial ovarian carcinoma. Clin Cancer Res 20:565-575. doi:10.1158/10780432.CCR-13-1271

14. Peng C, Liu HY, Zhou M et al (2007) BRD7 suppresses the growth of nasopharyngeal carcinoma cells (HNE1) through negatively regulating $\beta$-catenin and ERK pathways. Mol Cell Biochem 303:141-149. doi:10.1007/s11010-007-9466-x

15. Harte MT, O'Brien GJ, Ryan NM et al (2010) BRD7, a subunit of SWI/SNF complexes, binds directly to BRCA1 and regulates BRCA1-dependent transcription. Cancer Res 70:2538-2547. doi:10.1158/0008-5472.CAN-09-2089

16. Kaeser MD, Aslanian A, Dong M et al (2008) BRD7, a novel PBAF-specific SWI/SNF subunit, is required for target gene activation and repression in embryonic. J Biol Chem 283:3225432263. doi:10.1074/jbc.M806061200.PMCID

17. Peng C, Zhou J, Liu HY et al (2006) The transcriptional regulation role of BRD7 by binding to acetylated histone through bromodomain. J Cell Biochem 97:882-892. doi:10.1002/jcb.20645

18. Burrows AE, Smogorzewska A, Elledge SJ (2010) Polybromoassociated BRG1-associated factor components BRD7 and BAF180 are critical regulators of p53 required for induction of replicative senescence. Proc Natl Acad Sci USA 107:1428014285. doi:10.1073/pnas.1009559107

19. Heo J-I, Kim W, Choi KJ et al (2016) XIAP-associating factor 1, a transcriptional target of BRD7, contributes to endothelial cell senescence. Oncotarget 7:5118-5130. doi:10.18632/ oncotarget.6962

20. Dirkx AEM, oude Egbrink MGA, Castermans K et al (2006) Antiangiogenesis therapy can overcome endothelial cell anergy and promote leukocyte-endothelium interactions and infiltration in tumors. FASEB J 20:621-630. doi:10.1096/fj.05-4493com

21. Griffioen AW, Damen CA, Martinotti S et al (1996) Endothelial intercellular adhesion molecule- 1 expression is suppressed in human malignancies: the role of angiogenic factors. Cancer Res 56:1111-1117

22. Ades EW, Candal FJ, Swerlick RA et al (1992) HMEC-1: establishment of an immortalized human microvascular endothelial cell line. J Invest Dermatol 99:683-690

23. Fontijn R, Hop C, Brinkman HJ et al (1995) Maintenance of vascular endothelial cell-specific properties after immortalization with an amphotrophic replication-deficient retrovirus containing human papilloma virus 16 E6/E7 DNA. Exp Cell Res 216:199207. doi:10.1006/excr.1995.1025

24. van Beijnum JR, van der Linden E, Griffioen AW (2008) Angiogenic profiling and comparison of immortalized endothelial cells for functional genomics. Exp Cell Res 314:264-272. doi:10.1016/j.yexcr.2007.08.013

25. Thijssen VL, Hulsmans S, Griffioen AW (2008) The galectin profile of the endothelium: altered expression and localization in activated and tumor endothelial cells. Am J Pathol 172:545-553. doi:10.2353/ajpath.2008.070938

26. Thijssen VL, Brandwijk RJ, Dings RP, Griffioen AW (2004) Angiogenesis gene expression profiling in xenograft models to study cellular interactions. Exp Cell Res 299:286-293. doi:10.1016/j. yexcr.2004.06.014

27. Kzhyshkowska J, Rusch A, Wolf H, Dobner T (2003) Regulation of transcription by heterogeneous nuclear ribonucleoprotein E1B-AP5 is mediated by complex formation with the novel bromodomain containing protein BRD7. Biochem J 371:385-393. doi:10.1042/BJ20021281

28. Xu K, Xiong W, Zhou M et al (2016) Integrating ChIP-sequencing and digital gene expression profiling to identify BRD7 downstream genes and construct their regulating network. Mol Cell Biochem 411:57-71. doi:10.1007/s11010-015-2568-y

29. Zhou J, Ma J, Zhang BC et al (2004) BRD7, a novel bromodomain gene, inhibits G1-S progression by transcriptionally regulating some important molecules involved in ras/MEK/ERK and $\mathrm{Rb} /$ E2F pathways. J Cell Physiol 200:89-98. doi:10.1002/jcp.20013

30. Wu WJ, Hu KS, Chen DL et al (2013) Prognostic relevance of BRD7 expression in colorectal carcinoma. Eur J Clin Invest 43:131-140. doi:10.1111/eci.12024

31. Argos M, Kibriya MG, Jasmine F et al (2008) Genomewide scan for loss of heterozygosity and chromosomal amplification in breast carcinoma using single-nucleotide polymorphism arrays. Cancer Genet Cytogenet 182:69-74. doi:10.1016/j. cancergencyto.2008.01.001

32. Akino T, Hida K, Hida Y et al (2009) Cytogenetic abnormalities of tumor-associated endothelial cells in human malignant tumors. Am J Pathol 175:2657-2667. doi:10.2353/ajpath.2009.090202

33. Liu H, Zhang L, Niu Z et al (2008) Promoter methylation inhibits BRD7 expression in human nasopharyngeal carcinoma cells. BMC Cancer 8:253. doi:10.1186/1471-2407-8-253

34. Nowak-Sliwinska P, Segura T, Iruela-Arispe ML (2014) The chicken chorioallantoic membrane model in biology, medicine and bioengineering. Angiogenesis 17:779-804. doi:10.1007/ s10456-014-9440-7

35. Nowak-Sliwinska P, Weiss A, van Beijnum JR et al (2015) Photoactivation of lysosomally sequestered sunitinib after angiostatic treatment causes vascular occlusion and enhances tumor growth inhibition. Cell Death Dis 6:e1641. doi:10.1038/cddis.2015.4

36. Weiss A, Ding X, van Beijnum JR et al (2015) Rapid optimization of drug combinations for the optimal angiostatic treatment of cancer. Angiogenesis 18:233-244. doi:10.1007/s10456-015-9462-9

37. Nowak-Sliwinska P, Weiss A, van Beijnum JR et al (2012) Angiostatic kinase inhibitors to sustain photodynamic angio-occlusion. J Cell Mol Med 16:1553-1562. doi:10.1111/j.1582-4934.2011.01440.x

38. Tabruyn SP, Memet S, Ave P et al (2009) NF- $\kappa$ B activation in endothelial cells is critical for the activity of angiostatic agents. Mol Cancer Ther 8:2645-2654. doi:10.1158/1535-7163. MCT-09-0383

39. Kutmon M, Riutta A, Nunes N et al (2016) WikiPathways: capturing the full diversity of pathway knowledge. Nucleic Acids Res 44:D488-D494. doi:10.1093/nar/gkv1024

40. Zou Z, Huang B, Wu X et al (2014) Brd4 maintains constitutively active NF- $\mathrm{\kappa B}$ in cancer cells by binding to acetylated RelA. Oncogene 33:2395-2404. doi:10.1038/onc.2013.179 
41. Richmond A (2002) NF- $\kappa \mathrm{B}$, chemokine gene transcription and tumour growth. Nat Rev Immunol 2:664-674. doi:10.1038/nri887

42. Lee MYK, Wang Y, Vanhoutte PM (2010) Senescence of cultured porcine coronary arterial endothelial cells is associated with accelerated oxidative stress and activation of NFkB. J Vasc Res 47:287-298. doi:10.1159/000265563

43. Shelton DN, Chang E, Whittier PS et al (1999) Microarray analysis of replicative senescence. Curr Biol 9:939-945

44. Adler AS, Kawahara TLA, Segal E, Chang HY (2008) Reversal of aging by NF- $\kappa B$ blockade. Cell Cycle 7:556-559. doi:10.4161/ cc.7.5.5490

45. Zhao R, Liu Y, Wang $\mathrm{H}$ et al (2016) BRD7 plays an anti-inflammatory role during early acute inflammation by inhibiting activation of the NF- $\mathrm{KB}$ signaling pathway. Cell Mol Immunol. doi:10.1038/cmi.2016.31

46. Wiley MM, Muthukumar V, Griffin TM, Griffin CT (2015) SWI/ SNF chromatin-remodeling enzymes Brahma-related gene 1 (BRG1) and Brahma (BRM) are dispensable in multiple models of postnatal angiogenesis but are required for vascular integrity in infant mice. J Am Heart Assoc 4:e001972. doi:10.1161/ JAHA.115.001972

47. Singh AP, Foley JF, Rubino M et al (2016) Brg1 enables rapid growth of the early embryo by suppressing genes that regulate apoptosis and cell growth arrest. Mol Cell Biol 36:1990-2010. doi:10.1128/MCB.01101-15

48. Bultman SJ, Gebuhr TC, Pan H et al (2006) Maternal BRG1 regulates zygotic genome activation in the mouse. Genes Dev 20:1744-1754. doi:10.1101/gad.1435106

49. Chiu Y-H, Lee JY, Cantley LC (2014) BRD7, a tumor suppressor, interacts with $\mathrm{p} 85 \alpha$ and regulates PI3K activity. Mol Cell 54:193-202. doi:10.1016/j.molcel.2014.02.016

50. Sun J, Nie J, Hao B et al (2008) Ceap/BLOS2 interacts with BRD7 and selectively inhibits its transcription-suppressing effect on cellular proliferation-associated genes. Cell Signal 20:1151-1158. doi:10.1016/j.cellsig.2008.02.002

51. Briot A, Iruela-Arispe ML (2015) Blockade of specific notch ligands: a new promising approach in cancer therapy. Cancer Discov 5:112-114. doi:10.1158/2159-8290.CD-14-1501

52. Haghnegahdar H, Du J, Wang D et al (2000) The tumorigenic and angiogenic effects of MGSA/GRO proteins in melanoma. J Leukoc Biol 67:53-62

53. Gijsbers K, Gouwy M, Struyf S et al (2005) GCP-2/CXCL6 synergizes with other endothelial cell-derived chemokines in neutrophil mobilization and is associated with angiogenesis in gastrointestinal tumors. Exp Cell Res 303:331-342. doi:10.1016/j. yexcr.2004.09.027

54. Verbeke H, Struyf S, Berghmans N et al (2011) Isotypic neutralizing antibodies against mouse GCP-2/CXCL6 inhibit melanoma growth and metastasis. Cancer Lett 302:54-62. doi:10.1016/j. canlet.2010.12.013

55. Kim SW, Lee DW, Yu LH et al (2012) Mesenchymal stem cells overexpressing GCP-2 improve heart function through enhanced angiogenic properties in a myocardial infarction model. Cardiovasc Res 95:495-506. doi:10.1093/cvr/cvs224

56. van Beijnum JR, Buurman WA, Griffioen AW (2008) Convergence and amplification of toll-like receptor (TLR) and receptor for advanced glycation end products (RAGE) signaling pathways via high mobility group B1 (HMGB1). Angiogenesis 11:91-99. doi:10.1007/s10456-008-9093-5

57. Amin AR, Islam ABMMK (2014) Genomic analysis and differential expression of HMG and S100A family in human arthritis: upregulated expression of chemokines, IL- 8 and nitric oxide by HMGB1. DNA Cell Biol 33:550-565. doi:10.1089/dna.2013.2198

58. Lu J, Ye X, Fan F et al (2013) Endothelial cells promote the colorectal cancer stem cell phenotype through a soluble form of Jagged-1. Cancer Cell 23:171-185. doi:10.1016/j.ccr.2012.12.021 